\title{
Surfactant/ $\mathbf{I}_{2}$ / Water: An Efficient System for Deprotection of Oximes
}

\section{and Imines to Carbonyls Under Neutral Conditions in Water}

\author{
Pranjal Gogoi, Parasa Hazarika and Dilip Konwar* \\ Organic Chemistry Division, Regional Research Laboratory, Jorhat -785006, Assam, India
}

\section{Supporting Information}

\section{Table of Contents:}

General Information

Characterization data for compounds $3(\mathrm{a}-\mathrm{c})$

Characterization data for compounds $3(\mathrm{~d}-\mathrm{h})$

Characterization data for compounds 3(i-m)

Characterization data for compounds $3(\mathrm{n}-\mathrm{q})$
S2

S2

S3

S4

S5 


\section{General Information}

${ }^{1} \mathrm{H}$ NMR spectra were recorded on $60 \mathrm{MHz}$. Chemical shifts are expressed in $\delta$ units relative to tetramethylsilane (TMS) signal as internal reference in $\mathrm{CDCl}_{3}$. FTIR spectra were recorded in $\mathrm{CHCl}_{3}$ or on $\mathrm{KBr}$ pellets. Commercially available $\mathrm{I}_{2}$, Sodium dodecyl sulphate (SDS) were used directly and oximes and imines were prepared by following standard literature procedure. Column chromatography was performed on silica gel (60-120 mesh) using ethyl acetate and hexane as eluent.

\section{Characterization Data:}

3a. Cyclohexanone: According to the general procedure, cyclohexanone oxime gave cyclohexanone, $74.5 \mathrm{mg}$ (isolated yield; 76\%) as a liquid. ${ }^{1} \mathrm{H} \mathrm{NMR}\left(\mathrm{CDCl}_{3}, 60 \mathrm{MHz}\right): \delta 2.3-2.4(\mathrm{~m}$, 4H), 1.7-1.9 (m, 6H). FTIR $\left(\mathrm{CHCl}_{3}\right): 1715.4 \mathrm{~cm}^{-1}$. Anal. Calcd. For $\mathrm{C}_{10} \mathrm{H}_{10} \mathrm{O}$ (98.15): C, 73.45; H, 10.27. Found: C, 73.55; H, 10.21 .

3b. 6-Methoxy-1-tetralone: According to the general procedure, 6-Methoxy-1-tetralone oxime gave 6-methoxy-1-tetralone as a solid, M.p.78 ${ }^{0} \mathrm{C} ; 123 \mathrm{mg}$ (isolated yield; $\left.69.8 \%\right),{ }^{1} \mathrm{H}$ NMR $\left(\mathrm{CDCl}_{3}, 60\right.$ MHz) $\delta 7.6(\mathrm{~d}, 1 \mathrm{H}), 6.5(\mathrm{~d}, 1 \mathrm{H}), 6.3(\mathrm{~s}, 1 \mathrm{H}), 3.6(\mathrm{~s}, 3 \mathrm{H}), 1.7-2.1(\mathrm{~m}, 2 \mathrm{H}), 2.2-2.5(\mathrm{t}, 2 \mathrm{H}), 2.6-2.8(\mathrm{t}$, 2H); FTIR (KBr) $1606 \mathrm{~cm}^{-1}$. Anal. Calcd. For $\mathrm{C}_{11} \mathrm{H}_{12} \mathrm{O}_{2}$ (176.22): C, 74.98; H, 6.86. Found: C, $75.05 ; \mathrm{H}, 6.78$.

3c. Acetophenone: According to the general procedure, acetophenone oxime gave acetophenone as a liquid; $96 \mathrm{mg}$ (isolated yield; 80\%). ${ }^{1} \mathrm{H} \mathrm{NMR}\left(\mathrm{CDCl}_{3}, 60 \mathrm{MHz}\right) \delta 7.8-8.0(\mathrm{~m}, 2 \mathrm{H}), 7.3-7.6(\mathrm{~m}, 3 \mathrm{H})$, 2.57 (s, 3H); FTIR $\left(\mathrm{CHCl}_{3}\right) 1685 \mathrm{~cm}^{-1}$. Anal. Calcd. For $\mathrm{C}_{8} \mathrm{H}_{8} \mathrm{O}$ (120.15): C, 79.97; H, 6.71. Found: C, $79.80 ; \mathrm{H}, 6.58$. 
3d. 4-isobutylacetophenone: According to the general procedure, 4-isobutylacetophenone oxime gave 4-isobutyl acetophenone as a liquid; $137 \mathrm{mg}$, (isolated yield $77.8 \%$ ). ${ }^{1} \mathrm{H}$ NMR (60 MHz, $\left.\mathrm{CDCl}_{3}\right): \delta 0.81(\mathrm{~d}, 6 \mathrm{H}), 1.30-2.00(\mathrm{~m}, 1 \mathrm{H}), 2.40(\mathrm{~d}, 2 \mathrm{H}), 2.50(\mathrm{~s}, 3 \mathrm{H}), 6.90(\mathrm{~d}, 2 \mathrm{H}$, aromatic- $\mathrm{H}), 7.55(\mathrm{~d}$, 2H, aromatic-H); FTIR: $\left(\mathrm{CHCl}_{3}\right) 1682 \mathrm{~cm}^{-1}$. Anal. Calcd. For $\mathrm{C}_{12} \mathrm{H}_{16} \mathrm{O}$ (176.3): C, 81.77; H, 9.15. Found: C, 81.75; H, 9.20.

3e. Benzophenone: According to the general procedure, benzophenone oxime gave benzophenone as a solid, M.p. $48{ }^{0} \mathrm{C} ; 127 \mathrm{mg}$ (isolated yield; 69.8\%), ${ }^{1} \mathrm{H}$ NMR $\left(\mathrm{CDCl}_{3}, 60 \mathrm{MHz}\right) \delta$ 7.4-7.8 (m, $10 \mathrm{H}$ ); FTIR (KBr) $1665 \mathrm{~cm}^{-1}$. Anal. Calcd. For $\mathrm{C}_{13} \mathrm{H}_{10} \mathrm{O}$ (182.22): C, 85.69; H, 5.53. Found: $\mathrm{C}$, 85.51; H, 5.22.

3f. Benzaldehyde: According to the general procedure, benzaldoxime gave $92 \mathrm{mg}(86.8 \%$, isolated yield) of benzaldehyde as liquid, ${ }^{1} \mathrm{H}$ NMR $\left(\mathrm{CDCl}_{3}, 60 \mathrm{MHz}\right) \delta 10.2(\mathrm{~s}, 1 \mathrm{H})$, 7.5-7.9 (m, 5H); FTIR $\left(\mathrm{CHCl}_{3}\right) 1701 \mathrm{~cm}^{-1}$. Anal. Calcd. For $\mathrm{C}_{7} \mathrm{H}_{6} \mathrm{O}$ (106.12): C, 79.23; H, 5.70. Found: C, 79.50; H, 5.29. 3g. 4-Methoxybenzaldehyde: According to the general procedure, 4-methoxybenzaldoxime gave 4methoxybenzaldehyde, $123 \mathrm{mg}$ (isolated yield; 90.4\%) as a liquid; ${ }^{1} \mathrm{H} \mathrm{NMR}\left(\mathrm{CDCl}_{3}, 60 \mathrm{MHz}\right) \delta 9.87$ (s, 1H), $7.83(\mathrm{~d}, 2 \mathrm{H}), 7.0(\mathrm{~d}, 2 \mathrm{H}), 3.87(\mathrm{~s}, 3 \mathrm{H})$; FTIR $\left(\mathrm{CHCl}_{3}\right) 1684 \mathrm{~cm}^{-1}$. Anal. Calcd. For $\mathrm{C}_{8} \mathrm{H}_{11} \mathrm{O}_{2}$ (136.15): C, 70.58; H, 8.14. Found: C, 70.58; H, 8.23.

3h. 4-Nitrobenzaldehyde: According to the general procedure, 4-nitrobenzaldoxime gave $113 \mathrm{mg}$ (74.8\%, isolated yield) of 4-nitrobenzaldehyde as a solid, M.p.106-108 ${ }^{0} \mathrm{C} ;{ }^{1} \mathrm{H}$ NMR $\left(\mathrm{CDCl}_{3}, 60\right.$ MHz) $\delta 10.3(\mathrm{~s}, 1 \mathrm{H}), 8.3(\mathrm{~d}, 2 \mathrm{H}), 8.0(\mathrm{~d}, 2 \mathrm{H}) ;$ FTIR $(\mathrm{KBr}) 1680 \mathrm{~cm}^{-1}$. Anal. Calcd. For $\mathrm{C}_{7} \mathrm{H}_{5} \mathrm{NO}_{3}$ (151.12): C, 55.64; H, 3.33; N, 9.27. Found: C, 55.70; H, 3.27; N, 9.32. 
3i. $p$-Dimethylaminobenzaldehyde. According to the general procedure, $p$ dimethylaminobenzaldoxime gave $107 \mathrm{mg}$ (71.8\%, isolated yield) of $p$-dimethylaminobenzaldehyde as a solid, M.p. $74{ }^{0} \mathrm{C} ;{ }^{1} \mathrm{H} \mathrm{NMR}\left(\mathrm{CDCl}_{3}, 60 \mathrm{MHz}\right) \delta 9.70(\mathrm{~s}, 1 \mathrm{H}), 7.71(\mathrm{dd}, 2 \mathrm{H}), 6.69(\mathrm{dd}, 2 \mathrm{H})$, 3.05 (s, 6H); FTIR (KBr) $1672 \mathrm{~cm}^{-1}$. Anal. Calcd. For $\mathrm{C}_{9} \mathrm{H}_{11} \mathrm{NO}$ (149.19): C, 72.46; H, 7.43; N, 9.39. Found: C, 72.55; H, 7.42; N, 9.34.

3j. Vanillin. According to the general procedure, vanilline oxime gave $110 \mathrm{mg}(72.5 \%$, isolated yield) of vanilline as a solid, M.p.81-84 ${ }^{0} \mathrm{C} ;{ }^{1} \mathrm{H} \mathrm{NMR}\left(\mathrm{CDCl}_{3}, 60 \mathrm{MHz}\right) \delta 9.78(\mathrm{~s}, 1 \mathrm{H}), 7.02-7.4(\mathrm{~m}$, 3H), 6.47 (s, 1H), 3.93 (s, 3H); FTIR (KBr) $1696 \mathrm{~cm}^{-1}$. Anal. Calcd. For $\mathrm{C}_{8} \mathrm{H}_{8} \mathrm{O}_{3}$ (152.15): C, 63.15; H, 5.30. Found: C, 63.11; H, 5.28.

3k. Cinnamaldehyde: According to the general procedure, cinnamaldehyde oxime gave Cinnamaldehyde as a liquid; $96 \mathrm{mg}$ (isolated yield; $72.7 \%){ }^{1} \mathrm{H} \mathrm{NMR}\left(\mathrm{CDCl}_{3}, 60 \mathrm{MHz}\right) \delta 9.7(\mathrm{~d}, 1 \mathrm{H})$, 7.4-7.5 (m, 5H), 6.5-6.7 (m, 2H); FTIR $\left(\mathrm{CHCl}_{3}\right) 1681 \mathrm{~cm}^{-1}$. Anal. Calcd. For $\mathrm{C}_{9} \mathrm{H}_{8} \mathrm{O}$ (132.16): C, 81.79; H, 6.10. Found: C, 81.87; H, 6.20.

31. 2-Furfaldehyde: According to the general procedure, furfural oxime gave furfural as a liquid; 73 mg, (isolated yield $76 \%$ ). ${ }^{1} \mathrm{H}$ NMR $\left(60 \mathrm{MHz}, \mathrm{CDCl}_{3}\right): \delta 9.7(\mathrm{~s}, 1 \mathrm{H}), 7.7(\mathrm{~s}, 1 \mathrm{H}), 7.28(\mathrm{~s}, 1 \mathrm{H}), 6.6(\mathrm{~s}$, 1H); FTIR: $\left(\mathrm{CHCl}_{3}\right) 1682 \mathrm{~cm}^{-1}$. Anal. Calcd. For $\mathrm{C}_{5} \mathrm{H}_{4} \mathrm{O}_{2}$ (96.09): C, 62.50; H, 4.20. Found: $\mathrm{C}$, $62.11 ; \mathrm{H}, 4.27$.

3m. 1-Chloro-6-methoxy-3,4-dihydronaphthyl-2-carboxaldehyde: According to the general procedure, 1-Chloro-6-methoxy-3,4-dihydronaphthyl-2-carboxaldoxime gave 1-Chloro-6-methoxy3,4-dihydronaphthyl-2-carboxaldehyde as a solid, M.p. $91{ }^{0} \mathrm{C}$; $145 \mathrm{mg}$, (isolated yield $65 \%$ ). ${ }^{1} \mathrm{H}$ NMR (60 MHz, $\left.\mathrm{CDCl}_{3}\right): \delta 10.25(\mathrm{~s}, 1 \mathrm{H}), 7.85(\mathrm{dd}, 1 \mathrm{H}), 7.60(\mathrm{~d}, 1 \mathrm{H}), 6.76(\mathrm{~d}, 1 \mathrm{H}), 3.74(\mathrm{~s}, 3 \mathrm{H}), 2.50$ (m, 4H); FTIR: (KBr) $1672 \mathrm{~cm}^{-1}$. Anal. Calcd. For $\mathrm{C}_{12} \mathrm{H}_{11} \mathrm{O}_{2} \mathrm{Cl}$ (222.67): C, 64.73; H, 4.98. Found: C, 64.53; H, 5.12 . 
3n. 2-Pyridinecarboxaldehyde: According to the general procedure, 2-Pyridinecarboxaldoxime gave $76 \mathrm{mg}\left(71 \%\right.$, isolated yield) of 2-Pyridinecarboxaldehyde as a pale yellow liquid. ${ }^{1} \mathrm{H}$ NMR $\left(\mathrm{CDCl}_{3}, 60 \mathrm{MHz}\right) \delta 10.3(\mathrm{~s}, 1 \mathrm{H}), 8.7-8.8(\mathrm{~m}, 1 \mathrm{H})$, 7.8-8.0 (m, 2H), 7.5-7.6 (m, 1H); FTIR (KBr) $1715 \mathrm{~cm}^{-1}$. Anal. Calcd. For $\mathrm{C}_{6} \mathrm{H}_{5} \mathrm{NO}$ (107.11): C, 67.28; H, 4.71. Found: C, 67.25; H, 4.72.

3o. Benzaldehyde: According to the general procedure, benzylideneaniline gave $76 \mathrm{mg}(72 \%$, isolated yield) of benzaldehyde as liquid, ${ }^{1} \mathrm{H} \mathrm{NMR}\left(\mathrm{CDCl}_{3}, 60 \mathrm{MHz}\right) \delta 10.2(\mathrm{~s}, 1 \mathrm{H}), 7.5-8.0(\mathrm{~m}, 5 \mathrm{H})$; FTIR $\left(\mathrm{CHCl}_{3}\right) 1696 \mathrm{~cm}^{-1}$. Anal. Calcd. For $\mathrm{C}_{7} \mathrm{H}_{6} \mathrm{O}$ (106.12): C, 79.23; H, 5.70. Found: C, 79.50; H, 5.29 .

3p. p-Chlorobenzaldehyde. According to the general procedure, $\mathrm{N}-[(p-$ chlorophenyl)methylene]benzenamine gave $94 \mathrm{mg}$ (67\%, isolated yield) of $p$-chlorobenzaldehyde as a solid, M.p.47-50 ${ }^{0} \mathrm{C} ;{ }^{1} \mathrm{H}$ NMR $\left(\mathrm{CDCl}_{3}, 60 \mathrm{MHz}\right) \delta 9.8(\mathrm{~s}, 1 \mathrm{H}), 7.5-7.75(\mathrm{~m}, 4 \mathrm{H})$; FTIR $(\mathrm{KBr}) 1687$ $\mathrm{cm}^{-1}$. Anal. Calcd. For $\mathrm{C}_{7} \mathrm{H}_{5} \mathrm{OCl}$ (140.57): C, 59.81; H, 3.58. Found: C, 59.55; H, 3.72.

3q. $p$-Tolualdehyde. According to the general procedure, $\mathrm{N}-[(p-$ methyl)phenyl]methylene]benzenamine gave $82 \mathrm{mg}(68.2 \%$, isolated yield) of $p$-tolualdehyde as a liquid; ${ }^{1} \mathrm{H} \mathrm{NMR}\left(\mathrm{CDCl}_{3}, 60 \mathrm{MHz}\right) \delta 9.8(\mathrm{~s}, 1 \mathrm{H}), 7.7(\mathrm{dd}, 2 \mathrm{H}), 7.2(\mathrm{dd}, 2 \mathrm{H}), 2.4(\mathrm{~s}, 3 \mathrm{H}) ; \mathrm{FTIR}(\mathrm{KBr})$ $1690 \mathrm{~cm}^{-1}$. Anal. Calcd. For $\mathrm{C}_{8} \mathrm{H}_{8} \mathrm{O}$ (120.15): C, 79.97; H, 6.71. Found: C, 79.95; H, 6.62. 